\title{
Sporulation Culture of Spheroplasts Disturbs the Formation of Normal Spore Pairs in the Yeast Saccharomycodes ludwigii
}

\author{
Isamu Miyakawa ${ }^{1,3 *}$, Ryotaro Yagi $^{2}$, Kazuyoshi Sugihara ${ }^{2}$ and Tomomi Inai ${ }^{1}$ \\ ${ }^{1}$ Department of Environmental Science and Engineering, Graduate School of Science and Engineering, \\ Yamaguchi University, 1677-1 Yoshida, Yamaguchi-shi, Yamaguchi 753-8512, Japan \\ ${ }^{2}$ Department of Biology, Faculty of Science, Yamaguchi University, \\ 1677-1 Yoshida, Yamaguchi-shi, Yamaguchi 753-8512, Japan \\ ${ }^{3}$ Core Research for Evolutional Science and Technology (CREST), Japan Science and Technology Agency, \\ Gobancho, Chiyoda-ku, Tokyo 102-0076, Japan
}

Received July 29, 2015; accepted October 25, 2015

\begin{abstract}
Summary In Saccharomycodes ludwigii, two spores with opposite mating types are tethered together by a ledge at the poles of a lemon-shaped apiculate cell. In the present study, we performed sporulation culture of spheroplasts of $S$. ludwigii in order to investigate how cell shape affects the formation of spore pairs. Each spore pair in whole cells or in spheroplasts was isolated using a micromanipulator, and the colonies derived from the germination of spore pairs were tested for sporulation ability and mating type. In terms of the results, among 55 colonies derived from spore pairs in whole cells, only one colony was haploid and the other 54 colonies were diploid. In contrast, among 55 colonies derived from spores in spheroplasts, 10 colonies were haploid and the other 45 colonies were diploid. These results, together with microscopic observations, suggest that the lemon-shaped cell morphology is important for the formation of normal spore pairs.
\end{abstract}

Key words Yeast, Saccharomycodes ludwigii, Sporulation, Spheroplast, Ledge formation.

Saccharomycodes ludwigii is a lemon-shaped yeast that grows by bipolar budding. It produces four spores in an ascus, in which two spores with opposite mating types are tethered by a distinct ledge, as revealed by electron microscopy (Kreger-van Rij 1969). The two spores within a pair are normally of the opposite mating types (Yamazaki et al. 1976). Accordingly, the two spores within a pair germinate and mate with each other without mitotic divisions to return to diploid cells. The mechanisms for the tethering of spores by the ledge ensure the unique sexual reproduction of this yeast. Electron microscopic observation of freeze-fracture replicas showed that the ledge is a raised annulus (Simmons and Ahearn 1985).

S. ludwigii is a yeast suitable for cytological research of the sporulation process, owing to its large cell size and high sporulation ratio. In a previous study, we revealed for the first time the morphological changes in mitochondrial nucleoids and mitochondria during the meiosis and sporulation of S. ludwigii (Miyakawa et al. 2012). During meiotic prophase, mitochondria maintained their tubular structures, and their structures subsequently assembled around the dividing nuclei during first and second meiotic divisions. Finally, some of

\footnotetext{
*Corresponding author, e-mail: miyakawa@yamaguchi-u.ac.jp DOI: $10.1508 /$ cytologia.81.47
}

the mitochondria were incorporated into spores that are located at both ends of the cells. We also investigated the behavior of meiotic nuclei by both DAPI and SYBRGreen staining. Although the first meiotic division occurs in either direction, the second meiotic division only occurred along the long axis of the cells. In those studies, we suggested that the direction of second meiotic division resulted in the close contact of two spores with opposite mating types in each pole of cells (Miyakawa et al. 2012).

In Saccharomyces cerevisiae, sporulation culture of spheroplasts has been successfully performed, in which the dynamic behavior of mitochondrial nucleoids (mt-nucleoids) and mitochondria has been revealed (Miyakawa et al. 1984, 1994). The meiotic karyotype of bivalent chromosomes has also been determined in spheroplasts performing meiosis (Kuroiwa et al. 1984, 1986). In the present study, we performed the sporulation culture of spheroplasts in S. ludwigii. Our results suggest that the sporulation in spheroplasts disturbs the production of normal spore pairs with opposite mating types.

\section{Materials and methods}

Strains and sporulation culture of whole cells

A diploid strain of S. ludwigii Hansen NBRC1721 (mating type a/ $\alpha$ ), and two haploid strains, NBRC1722 
(mating type a) and NBRC1723 (mating type $\alpha$ ), were used. The diploid strain was precultured on a YPD slant (1\% yeast extract, $2 \%$ peptone, $2 \%$ glucose) overnight. Cells were inoculated in $100 \mathrm{~mL}$ of modified Burkholder's medium supplemented with $3.6 \%(\mathrm{v} / \mathrm{v})$ tomato extract at an initial cell concentration of $\mathrm{OD}_{660}=0.01$, and cultured aerobically at $30^{\circ} \mathrm{C}$ for $30 \mathrm{~h}$ (Miyakawa et al. 2012). Cells that reached stationary phase at $30 \mathrm{~h}$ were harvested by centrifugation, washed with sterile distilled water, inoculated in $100 \mathrm{~mL}$ of sporulation medium $(0.98 \%$ potassium acetate, $1 \%$ glucose, $0.25 \%$ yeast extract) at a cell concentration of $3 \times 10^{6}$ cells $/ \mathrm{mL}$, and cultured aerobically with reciprocal shaking at $25^{\circ} \mathrm{C}$ for more than $60 \mathrm{~h}$ (McClary et al. 1959, Yamazaki et al. 1976, Miyakawa et al. 2012). More than 300 cells were counted to determine the stages of meiotic division and the sporulation ratio. The two haploid strains were used to determine the mating type of cells derived from the germination of spores.

\section{Spheroplast formation and sporulation culture of the spheroplasts}

Cells that reached stationary phase at $30 \mathrm{~h}$ of culture were harvested by centrifugation, washed with sterile distilled water, and inoculated in the sporulation medium as described above. After 30 -h sporulation culture, cells at meiotic prophase were harvested from $100-\mathrm{mL}$ sporulation culture and washed twice with sterile distilled water. Cells were suspended in $100 \mathrm{~mL}$ of SP buffer (0.8 M sorbitol, $50 \mathrm{mM}$ K-phosphate buffer $\mathrm{pH} 7.5)$ supplemented with $0.4 \mathrm{M} \beta$-mercaptoethanol and incubated for $30 \mathrm{~min}$ at $30^{\circ} \mathrm{C}$. After washing with $100 \mathrm{~mL}$ of SP buffer, cells were suspended in $100 \mathrm{~mL}$ of SP buffer that contained Zymolyase 20 T (Seikagaku Kogyo Co., Ltd., Tokyo, Japan; final concentration of $0.5 \mathrm{mg} \mathrm{mL}^{-1}$ ). Spheroplast formation was checked every $5 \mathrm{~min}$ using a phase-contrast microscope, and incubation was continued for up to $30 \mathrm{~min}$ at $30^{\circ} \mathrm{C}$. When cells were completely converted to spheroplasts with a round cell shape, the spheroplasts were collected, washed twice with SP buffer, and finally suspended in $100 \mathrm{~mL}$ of fresh sporulation medium prepared for spheroplasts $(0.8 \mathrm{M}$ sorbitol, $0.98 \%$ potassium acetate, $0.25 \%$ yeast extract). Sporulation culture was further continued for $60 \mathrm{~h}$ at $25^{\circ} \mathrm{C}$ with reciprocal shaking.

\section{Dispersion and isolation of spores}

Whole cells were harvested by centrifugation after sporulation culture for $60 \mathrm{~h}$. Cells were treated with Zymolyase $20 \mathrm{~T}$ to form spheroplasts as described above. The spheroplasts were harvested by centrifugation and washed twice with distilled water to disrupt the spheroplasts by osmotic shock. Spores were dispersed with a sonicator (Kubota UP-50H) at an amplitude of $80 \%, 0.5$ cycles, and 30 cycles on ice. On the other hand, spheroplasts were collected after sporulation culture by cen- trifugation, washed with sterile distilled water, and then dispersed with a sonicator as described above.

The dispersed spore pairs were spread on a YPD plate and each pair of spores was isolated using a micromanipulator. The isolated spore pairs were cultured on YPD plates for two days at $30^{\circ} \mathrm{C}$ to complete germination, mating, and vegetative growth. Cells from each colony were suspended once in $0.1 \mathrm{~mL}$ of sterile distilled water and $3 \mu \mathrm{L}$ of the cell suspension was spotted on modified Burkholder's medium plates and cultured for $24 \mathrm{~h}$ at $30^{\circ} \mathrm{C}$. Cells from each colony on the Burkholder's medium plates were again suspended in $0.1 \mathrm{~mL}$ of sterile distilled water and $3 \mu \mathrm{L}$ of the cell suspension was spotted on sporulation culture plates and cultured for three days at $25^{\circ} \mathrm{C}$. Cells from each colony on the sporulation culture plates were observed by phase-contrast microscopy for the formation of spores. The sporulation ratio of each diploid colony was usually more than $10 \%$.

In order to determine the mating type, cells from colonies that did not form spores on sporulation medium plates were mixed with either NBRC1722 (mating type a) or NBRC1723 (mating type $\alpha$ ) cells on YPD plates overnight at $30^{\circ} \mathrm{C}$; then, cells were suspended in $0.1 \mathrm{~mL}$ of sterile distilled water and $3 \mu \mathrm{L}$ of the cell suspension was spotted on modified Burkholder's medium agar plates and cultured for $24 \mathrm{~h}$ at $30^{\circ} \mathrm{C}$. Cells from each colony on the Burkholder's medium plates were again suspended in $0.1 \mathrm{~mL}$ of sterile distilled water and $3 \mu \mathrm{L}$ of the cell suspension was spotted on sporulation culture plates and cultured for $3 \mathrm{~d}$ at $25^{\circ} \mathrm{C}$. Cells from each colony on the sporulation culture plates were observed by phase-contrast microscopy for the formation of spores. Cells that formed spores by mixing with NBRC1722 (mating type a) cells but not with NBRC1723 (mating type $\alpha$ ) cells were determined to be of mating $\alpha$, and vice versa.

For the observation of germinating cells, spores isolated from both whole cells and from spheroplasts were dispersed by sonication, inoculated in YPD medium at $\mathrm{OD}_{660}=0.3$, and cultured for up to $9 \mathrm{~h}$ with shaking at $30^{\circ} \mathrm{C}$. The percentage of cell types was obtained by observing 300 cells.

\section{Microscopy}

Cells were fixed with $5 \%$ glutaraldehyde for $90 \mathrm{~min}$ at room temperature by the direct addition of $1 / 4$ volume of $25 \%$ glutaraldehyde solution to the culture medium. After three washes with NS buffer, cells were suspended with a small volume of NS buffer, and used for phasecontrast and 4',6-diamidino-2-phenylindole (DAPI) staining microscopy (Miyakawa et al. 1984, 2012). All observations were performed with an epifluorescence microscope (Model BX-53, OLYMPUS Optical Co., Ltd.) equipped with a digital camera (Nikon DIGITAL SIGHT DS-Qi1Mc). 


\section{Results}

Sporulation culture of whole cells and spheroplasts

The progress of meiotic division and the sporulation ratio of whole cells were observed by DAPI staining and phase-contrast microscopy (Fig. 1). The timing of sporulation was similar to that described in a previous study (Miyakawa et al. 2012). The first meiotic division began after $30 \mathrm{~h}$ of the culture and the second meiotic division immediately followed it. Mature asci normally contained two pairs of spores at the pole of cells (Fig. 2A). The final sporulation ratio reached about $50 \%$.

Spheroplasts are fragile and tend to be destroyed by long-term cultivation, even in culture medium supplemented with osmotic stabilizer. As the sporulation of $S$. cerevisiae was completed within $24 \mathrm{~h}$ after inoculation of the cells, spheroplasts of stationary-phase cells could

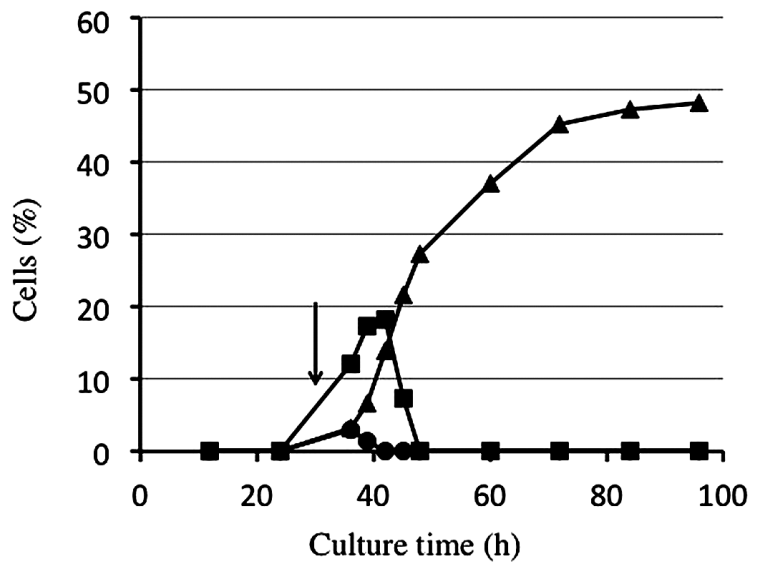

Fig. 1. Timing of meiosis and sporulation in S. ludwigii. Percentages of cells are shown. $\mathbf{O}$ : cells with two nuclei, $\mathbf{\square}$ : cells with four nuclei, $\boldsymbol{\Delta}$ : mature asci. An arrow at 30 -h culture indicates the timing of harvesting of cells for spheroplasting. be inoculated in the sporulation medium at the beginning of the culture in a previous study (Miyakawa et al. 1984). However, in the case of $S$. ludwigii, it took much longer, namely, up to $60 \mathrm{~h}$, to complete sporulation. Our preliminary experiments showed that almost all spheroplasts were disrupted until the onset of meiosis and sporulation, when spheroplasts were inoculated at the onset of the culture. Therefore, we used a modified method, in which whole cells were cultured for $30 \mathrm{~h}$ in sporulation medium, harvested once and converted to spheroplasts, and again inoculated in fresh sporulation medium prepared for spheroplasts. For the sporulation of spheroplasts, glucose was eliminated from the sporulation medium because the addition of glucose inhibited the sporulation of spheroplasts and induced their enlargement. Sorbitol was added to the sporulation medium at $0.8 \mathrm{M}$ as an osmotic stabilizer (Miyakawa et al. 1984). Ascospores that were formed in spheroplasts are shown in Fig. 2B. Although significant numbers of spheroplasts were disrupted during sporulation culture even in these conditions, ascospores were obtained at levels sufficient for isolation using a micromanipulator. Ascospores that were formed in both whole cells and spheroplasts were dispersed into spore pairs by sonication (Fig. 2C, D).

\section{Microscopic observation of germinating spores}

Spore pairs that were isolated from ascospores of whole cells were inoculated in YPD medium for germination. A pair of spores (Fig. 3A) germinated by protruding a short tube from each spore at $2 \mathrm{~h}$ (Fig. 3B), and then two germination tubes fused with each other at $4 \mathrm{~h}$ (Fig. 3C). The fused germination tubes gradually thickened and a new bud appeared at the apical end of the zygote at $9 \mathrm{~h}$ (Fig. 3D). Among germinating spores, a small number of spore pairs had only one germination tube
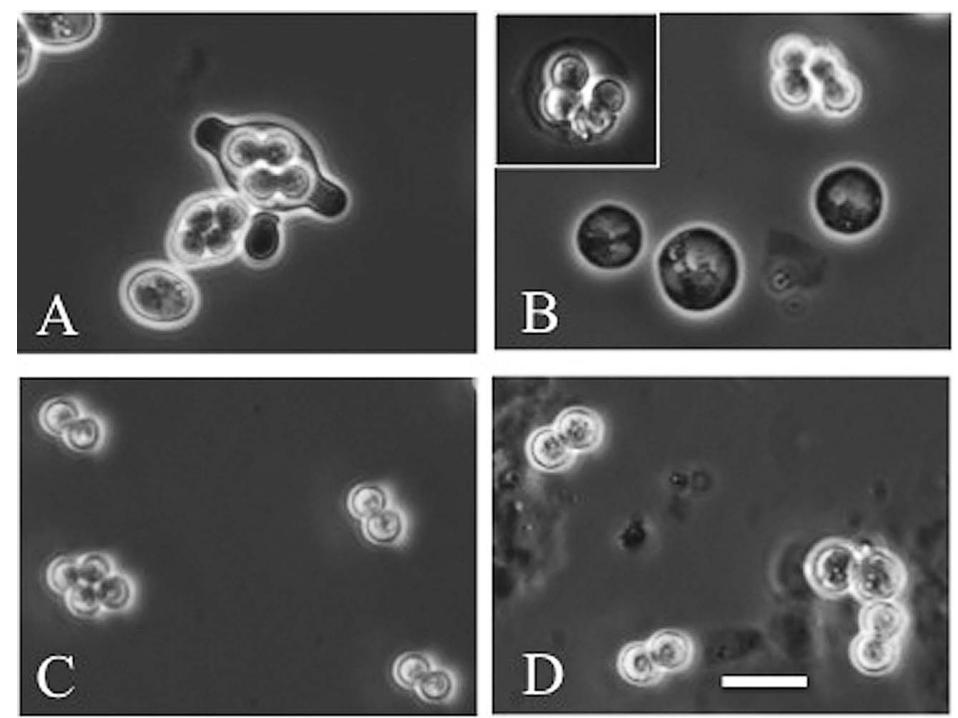

Fig. 2. Phase-contrast photomicrographs of sporulating cells and the isolated spore pairs. (A) Ascospores formed in whole cells by 60 -h sporulation culture. (B) Ascospores formed by 60-h sporulation culture of spheroplasts. Upper left photograph in B shows four spores in an ascus. (C) Spore pairs from whole cells that were dispersed by sonication. (D) Spore pairs from spheroplasts that were dispersed by sonication. Bar $10 \mu \mathrm{m}$. 

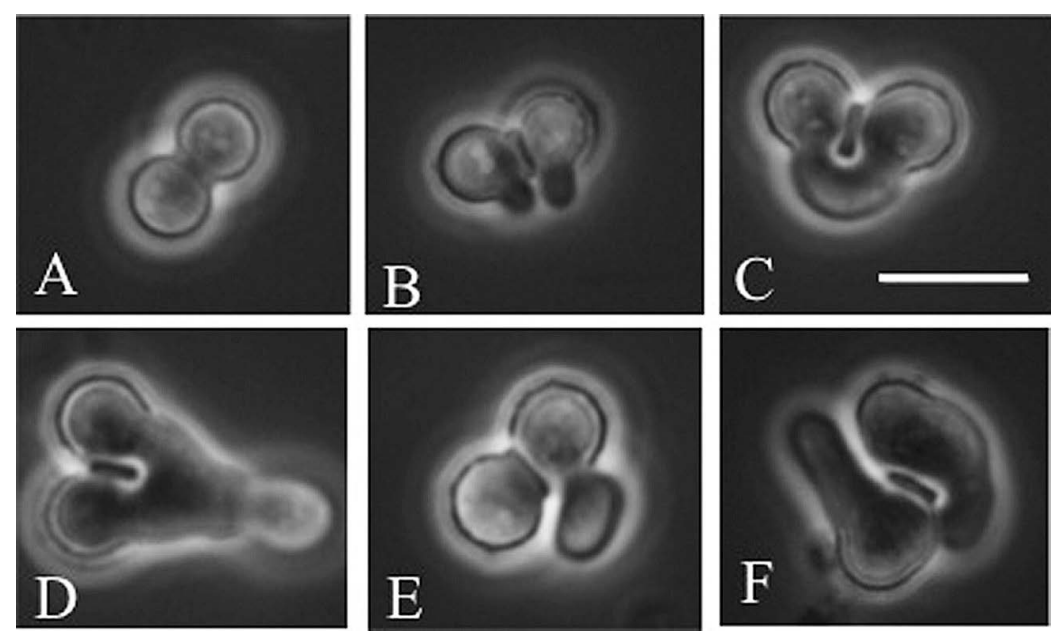

Fig. 3. Phase-contrast photomicrographs of germinating spores. (A) A pair of spores isolated from an ascus. (B) A pair of spores with small germinating tubes on both spores at 2-h culture. (C) A pair of spores with fused germination tubes at 4-h culture. (D) A zygote at 9-h culture. (E) A pair of spores with one germination tube on either spore at 9-h culture. (F) A pair of spores with two germination tubes but that do not mate at 9-h culture. Bar $5 \mu \mathrm{m}$.

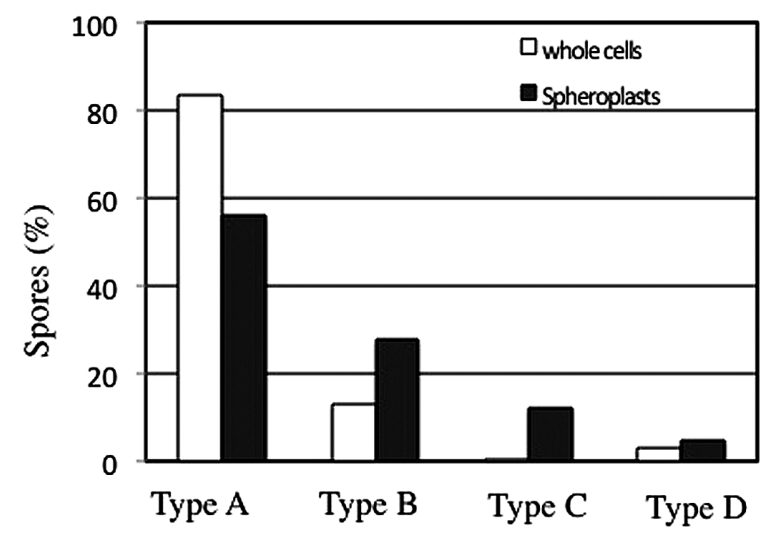

Fig. 4. Classification of germinating spore pairs. The morphology of spore pairs at 9-h germination culture was classified into four types. Type A: spore pairs that mated to make a zygote (Fig. 3D); Type B: spore pairs with one germination tube on either spore (Fig. 3E); Type C: spore pairs with two germination tubes on both spores but that do not mate (Fig. 3F); Type D: spore pairs that do not germinate.

on either spore (Fig. 3E). In addition, there were a small number of spore pairs that extended germination tubes from both spores but did not mate (Fig. 3F). We classified the morphology of germinating spores at 9-h culture into four types (Fig. 4). Among spores derived from whole cells, $83.7 \%$ of spores mated with each other (Fig. 4 , Type A), but $13 \%$ of spore pairs had one germinating tube on either spore (Fig. 4, Type B). The ratio of spore pairs that had two germination tubes but did not mate was $0.3 \%$ (Fig. 4, Type C). Three percent of spore pairs did not germinate even after $9 \mathrm{~h}$ of culture (Fig. 4, Type D). In contrast, the ratio of spore pairs that mated at $9 \mathrm{~h}$ of culture was decreased to $56 \%$ in spores derived from sporulation culture of spheroplasts (Fig. 4, Type A). Instead, the ratios of spore pairs of Type B, Type C, and Type D increased to $27.6,12$, and $4.6 \%$, respectively.
Table 1. Ploidy and mating type of cells derived from spore pairs.

\begin{tabular}{lcccc}
\hline \hline \multirow{2}{*}{ Colonies } & \multicolumn{3}{c}{ Mating type } \\
\cline { 2 - 4 } & $\mathrm{a} / \alpha$ & $\mathrm{a}$ & $\alpha$ \\
\hline Colonies derived from spore pairs in whole cells & 54 & 1 & 0 \\
Colonies derived from spore pairs in spheroplasts & 45 & 5 & 5 \\
\hline
\end{tabular}

\section{Isolation of spore pairs}

The spore pairs were isolated using a micromanipulator and then cultured on YPD plates. Cells derived from the germination of each spore pair were grown once on modified Burkholder's medium plates for $24 \mathrm{~h}$, transferred to sporulation medium plates, and cultured for $3 \mathrm{~d}$ at $25^{\circ} \mathrm{C}$. The sporulation of cells was tested by microscopy and the colonies that did not form spores were tested to determine the mating type by crossing with tester haploid strains. The sporulation ability of cells refers to the diploidization of cells by the mating of a pair of spores. In terms of the results, 54 of 55 colonies derived from spore pairs in whole cells were diploid and only one colony was haploid with mating type a (Table 1). In contrast, 10 colonies among 55 colonies derived from spore pairs in spheroplasts were haploid and the other 45 colonies were diploid. Five colonies among the 10 haploid colonies showed mating type a, and the other five colonies mating type $\alpha$ (Table 1).

\section{Discussion}

The life cycle of $S$. ludwigii is schematically shown in Fig. 5. In lemon-shaped whole cells, the second meiotic division occurs along the long axis of the cells irrespective of the direction of the first meiotic division. The spore wall is assembled around four nuclei and the ledges appear between neighboring spores to tether them. Consequently, spore pairs with opposite mating 

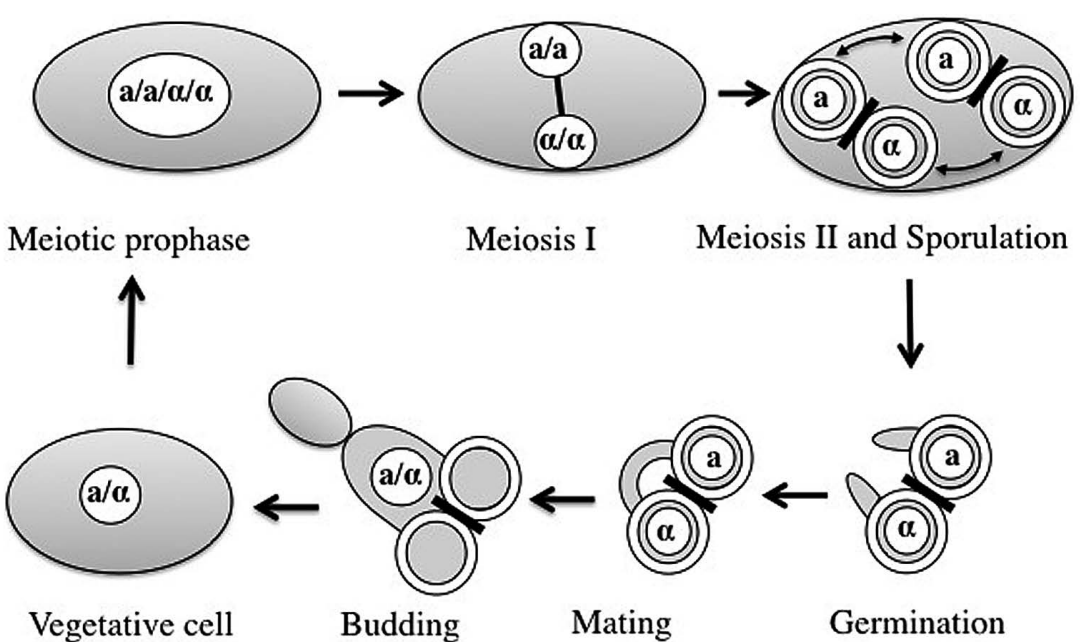

Fig. 5. A schematic model of the life cycle of S. ludwigii.

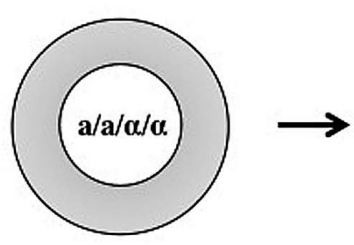

Meiotic prophase

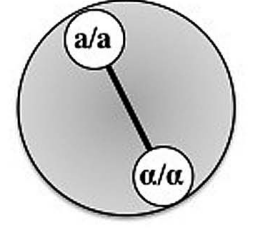

Meiosis I

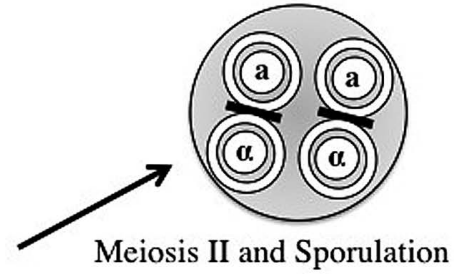

Meiosis II and Sporulation
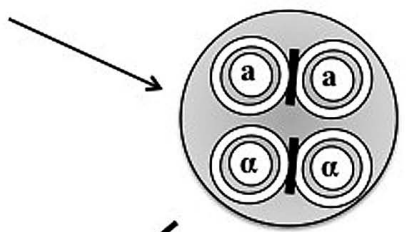
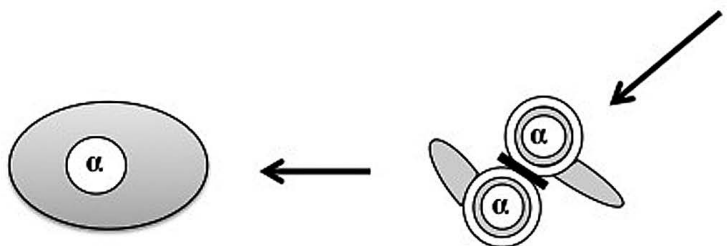

Fig. 6. A schematic model of the meiosis and sporulation in spheroplasts. Cell polarity of long and short axes will be lost in round cells like spheroplasts, in which meiotic division will occur in either direction. As a result, the occurrence of spore pairs with the same mating type will increase.

types were formed at each end of cells. The spore pairs germinate and mate with each other, and then a new bud emerges at the apical end of the zygote to return to diploid vegetative cells. Therefore, ledge formation between spores is a key determinant of the production of diploid cells in the unique sexual cycle of this yeast.

There are two possible explanations about how ledges are formed between spores. First, ledges may be formed between two spores of the opposite mating type by discriminating the mating type of spores. Second, ledges may be formed between spores that are in close contact, irrespective of their mating types. As shown in Table 1, many more haploid colonies were obtained from the germination of spore pairs produced in spheroplasts than those in whole cells. This suggests that the number of spore pairs with the same mating type increased in spheroplasts.

The results of microscopic observations do not com- pletely coincide with the results of ploidy of cells derived from spore pairs (Table 1, Fig. 4). Whereas the ratio of haploid colonies was $1.9 \%(1 / 55)$ among colonies derived from spore pairs of whole cells, $13 \%$ of spore pairs had only one germination tube on either spore at 9-h culture (Fig. 4). This suggests that almost all spore pairs with one germination tube at 9-h culture will finally fuse with each other to return to a diploid form after 48 -h culture. The ratio of spore pairs with one germination tube increased to $28 \%$ in the population of spores produced in spheroplasts. Estimating from the observations described above, a significant population of these spore pairs with one germination tube will also return to a diploid form after 48-h culture, and the other spore pairs that did not mate will produce haploid vegetative cells. It should be noted that $12 \%$ of spore pairs produced in spheroplasts had germination tubes on both spores but did not mate with each other. The ratio of this type of 
spore pairs was only $0.3 \%$ in a population of spores produced in whole cells. Therefore, it is likely that this type of spore pair from spheroplasts (Fig. 4, Type C) has the same mating type and produces haploid colonies. Taking these findings together with the morphological classification of germinating spores (Fig. 4) and the ploidy of colonies derived from spheroplasts (Table 1), our results suggest that at least several colonies among 10 haploid colonies are derived from spore pairs of the same mating type. It is also possible that other haploid colonies are derived from the germination of a single spore of spore pairs.

The production of spore pairs with the same mating type is schematically shown in Fig. 6. Apiculate cells are converted to round ones by Zymolyase treatment and lose cell polarity of long and short axes. Within those cells, spores of the same mating type will have more chance to be in close contact with each other. Consequently, an interspore structure, a ledge, may be produced between spores of the same mating type. Our observations support the idea that the ledges are formed between closely contacted spores irrespective of the mating type. Therefore, the lemon-shaped morphology of cells will be important for the production of spore pairs, each of which has an opposite mating type.

The presence of interspore bridges has been found between spores of $S$. cerevisiae by transmission and scanning electron microscopy (Coluccio and Neiman 2004, Neiman 2011). In the case of $S$. cerevisiae, the interspore bridges connect four spores, so that the wild-type spores remain in groups of four spores even after digestion of the ascus wall with Zymolyase. It has been shown that chitin or chitosan is required for bridge construction. Four spores of $S$. cerevisiae are easily dispersed by sonication, but spore pairs of $S$. ludwigii cannot be separated by this treatment. This implies that the ledges of $S$. ludwigii are rather more robust structures than the interspore bridges in $S$. cerevisiae. Intratetrad mating is widespread among fungi and is an important means for their sexual reproduction, in which fertilization can occur via the fusion of two products of the same meiosis (Zakharov 2005, Knop 2006). Further studies on $S$. ludwigii will provide an interesting model to uncover the mechanisms of ledge formation and intratetrad mating.

Acknowledgements

This work was partly supported by JSCP KAKENHI Grant Number 15K07168, by CREST, and by MEXT TOKUBETSUKEIHI to Isamu Miyakawa, Yamaguchi University.

\section{References}

Coluccio, A. and Neiman, A. M. 2004. Interspore bridges: A new feature of the Saccharomyces cerevisiae spore wall. Microbiology 150: 3189-3196.

Knop, M. 2006. Evolution of the hemiascomycete yeasts: On life styles and the importance of inbreeding. BioEssays 28: 696-708.

Kreger-van Rij, N. J. W. 1969. A new feature of the ascospores of Saccharomycodes ludwigii Hansen. Can. J. Microbiol. 15: 823-825.

McClary, D. O., Nulty, W. L. and Miller, G. R. 1959. Effect of potassium versus sodium in the sporulation of Saccharomyces. J. Bacteriol. 78: 362-368.

Miyakawa, I., Aoi, H., Sando, N. and Kuroiwa, T. 1984. Fluorescence microscopic studies of mitochondrial nucleoids during meiosis and sporulation in the yeast, Saccharomyces cerevisiae. J. Cell Sci. 66: 21-38.

Miyakawa, I., Higo, K., Osaki, F. and Sando, N. 1994. Double staining of mitochondria and mitochondrial nucleoids in the living yeast during the life cycle. J. Gen. Appl. Microbiol. 40: 1-14.

Miyakawa, I., Nakahara, A. and Ito, K. 2012. Morphology of mitochondrial nucleoids, mitochondria, and nuclei during meiosis and sporulation of the yeast, Saccharomycodes ludwigii. J. Gen. Appl. Microbiol. 58: 43-51.

Neiman, A. M. 2011. Sporulation in the budding yeast Saccharomyces cerevisiae. Genetics 189: 737-765.

Simmons, R. B. and Ahearn, D. G. 1985. Ascospore ornamentation in Saccharomycodes ludwigii. Mycologia 77: 660-662.

Yamazaki, T., Ohara, Y. and Oshima, Y. 1976. Rare occurrence of the tetratype tetrads in Saccharomycodes ludwigii. J. Bacteriol. 125: 461-466.

Zakharov, I. A. 2005. Intratetrad mating and its genetic and evolutionary consequences. Russ. J. Genet. 41: 402-411. 